Cipango $\begin{aligned} & \text { Cipango } \\ & \text { Cahiers d'études japonaises }\end{aligned}$

15 | 2008

Guerre, colonialisme et commémoration

\title{
Nationalisme culturel et mouvement démocratique : Discours et participations politiques du Seikyōsha (1887-1898
}

Morvan Perroncel

\author{
(2) OpenEdition \\ Journals \\ Édition électronique \\ URL : https://journals.openedition.org/cipango/172 \\ DOI : 10.4000/cipango. 172 \\ ISSN : 2260-7706 \\ Éditeur \\ INALCO \\ Édition imprimée \\ Date de publication : 1 janvier 2008 \\ Pagination : 301-306 \\ ISBN : 978-2-85837-177-4 \\ ISSN : $1164-5857$
}

Référence électronique

Morvan Perroncel, « Nationalisme culturel et mouvement démocratique : Discours et participations politiques du Seikyōsha (1887-1898 », Cipango [En ligne], 15 | 2008, mis en ligne le 14 novembre 2011, consulté le 30 juin 2021. URL : http://journals.openedition.org/cipango/172 ; DOI : https://doi.org/ $10.4000 /$ cipango. 172

Ce document a été généré automatiquement le 30 juin 2021.

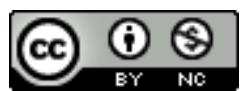

Cipango est mis à disposition selon les termes de la Licence Creative Commons Attribution - Pas d'Utilisation Commerciale 4.0 International. 


\title{
Nationalisme culturel et mouvement démocratique : Discours et participations politiques du Seikyōsha (1887-1898
}

\author{
Morvan Perroncel
}

\section{RÉFÉRENCE}

Nationalisme culturel et mouvement démocratique : Discours et participations politiques du Seikyōsha (1887-1898), Mémoire de Master 2 soutenu par Morvan Perroncel (Paris 7Diderot, 2008), sous la direction de A. Horiuchi et C. Hamon.

1 Cercle d'intellectuels fondé au début de la troisième décennie de Meiji, Seikyōsha 政教 社 est souvent présenté comme le meilleur exemple d'une réaction à l'occidentalisme des années 1880. On le mentionne généralement pour sa défense du "génie » ou de « l'excellence nationale» (kokusui shugi 国粋主義), exprimée à travers la revue Nihonjin (Les Japonais), dont le premier numéro parut en avril 1888. Ses représentants les plus connus sont le géographe Shiga Shigetaka (1863-1927) et le philosophe Miyake Setsurei (1860-1945), les deux seuls membres du groupe de départ qui aient continué d'animer la revue après 1892. Seikyōsha est en outre associé au quotidien Nihon, que Kuga Katsunan (1857-1907) créa en février 1889, avec lequel il forma le courant « nipponiste ».

Qu'on y discerne les prémices de l'ultranationalisme des années 1930 ou qu'on y voie, avec Maruyama Masao, la formulation d'un "nationalisme sain » attaché à la défense des droits du peuple, c'est toujours sur cet aspect du discours qu'on met l'accent, sans constater que les articles ou éditoriaux de Nihonjin n'ont finalement que peu traité de la doctrine à laquelle le nom de Seikyōsha reste lié, ou que la plupart des contributeurs n'ont même jamais précisé leur position sur le kokusuishugi. 
3 Il n'est pas dû au hasard que la revue ait été créée en 1888, alors que l'opposition libérale reprenait vigueur, mais on aurait tort de croire que la contestation des nouveaux projets de révision des traités inégaux qui l'accompagna dans les années 1887-1889 fut décisive dans le cas de Seikyōsha. Au printemps et à l'été de 1889, après que le contenu des traités négociés par ōkuma eut été révélé, Nihonjin se montra particulièrement modérée dans ses critiques, se réjouissant surtout du large intérêt suscité par la question, et invoquant finalement davantage le fait que l'opinion publique se montrait dans l'ensemble défavorable à la solution d'ōkuma, plutôt que tel ou tel argument particulier contre celle-ci. Cette attitude fut encore celle de Seikyōsha dans les mois qui précédèrent la Guerre sino-japonaise. Tout en se joignant au mouvement de contestation de la politique extérieure et en attaquant les compromis consentis en vue d'obtenir le recouvrement de la souveraineté douanière, il s'en prit d'abord et avant tout au refus du gouvernement de tenir compte de l'opinion, notamment de l'opposition parlementaire (la Chambre basse ayant été dissoute en décembre 1893 et en juin 1894).

Il est toutefois surprenant de constater que Seikyōsha n'a guère cherché à établir un lien théorique très clair entre ce libéralisme et le versant de son discours qui lui était plus propre, l'affirmation de l'originalité japonaise.

De tous les intervenants de Seikyōsha, Shiga Shigetaka est celui qui a le plus explicité cette idée. Ses premiers articles, où il appelait à la "préservation du génie national » (kokusui hozon shugi), s'appuyaient d'abord sur un raisonnement économique: l'occidentalisation de l'archipel, même une occidentalisation intégrale, était certes un moyen théoriquement concevable pour faire atteindre au Japon le niveau de civilisation des pays les plus avancés, mais cette voie était aberrante économiquement, supposant qu'on reparte de rien au lieu de profiter du développement déjà accompli. Or ce développement était intrinsèquement lié à l'environnement japonais, dont il tirait à la fois sa force et une originalité. Bien qu'elles aient dû emprunter leur matériau à des cultures antérieures, toutes les cultures de l'Europe avaient grandi à partir d'un fonds original et particulier. Comparant le développement des pays et des peuples à celui des êtres vivants, Shiga invoquait alors également la biologie.

$6 \quad$ Il se gardait en revanche de préciser en quoi pourrait consister ce qu'il appelait kokusui (vraisemblablement un mot inventé par lui-même) et insistait plutôt sur l'idée que cette excellence en devenir était portée par l'ensemble des Japonais, par la nation («Yamato-minzoku» ou «Nihon-kokumin»), manifestement plus fondamentale que son organisation en Etat, dont il ne parlait pas. On peut trouver cette affirmation de la nation cohérente avec le principe de responsabilité gouvernementale, mais on peut aussi noter que, tout en faisant du génie national le principe d'une unité de l'archipel et de sa population, Shiga ne lui donnait donc pas pour vocation de produire des institutions également originales.

7 Le seul du groupe qui ait tenté de prolonger ou reprendre la réflexion à partir de cette idée de kokusui est Kikuchi Kumatarō (1860-1904), qui s'efforça justement faire le lien avec les institutions politiques. Selon lui, le génie japonais tenait essentiellement dans les sentiments de la nation pour la Maison impériale. L'histoire montrait cependant que l'implication de la Maison impériale dans le gouvernement du pays mettait en péril cet attachement, de sorte qu'il valait mieux la décharger de toute responsabilité dans les décisions de l'exécutif, comme l'avait autrefois permis l'institution du bakufu. L'idée, faut-il remarquer, n'était pas nouvelle. Fukuzawa Yukichi avait écrit à peu près la 
même chose au milieu des années 1870. Kikuchi ne faisait en vérité que l'adapter au thème du génie national.

8 Un court éditorial non signé paru en mai 1889 annonça que la doctrine de Seikyōsha serait désormais «l'expression » (kenshō) du génie national, et non plus seulement sa préservation, mais on n'y faisait pas allusion à la Maison impériale, et peu semblait avoir été retenu de Shiga, sinon une sorte de pragmatisme. Aucune position commune ne se dégageait vraiment. Il ne fut d'ailleurs plus question du kokusui shugi par la suite. La position d'un autre membre, Inoue Enryō (1858-1919), qui voyait dans le bouddhisme le cœur de l'originalité japonaise et le meilleur de son avenir, apparaît même plus difficile encore à concilier avec celles de Shiga et Kikuchi.

9 Au-delà de ces divergences, on peut caractériser le discours de Seikyōsha dans son ensemble en disant qu'il a consisté à affirmer que le Japon et les Japonais avaient un destin original et que cela ne tenait pas essentiellement au fait que le Japon était un Etat. Dans toutes ses variantes (Shiga, Inoue, Kikuchi ou Miyake), on retrouve bien ce trait caractéristique du nationalisme, au sens le plus étroit, l'idée selon laquelle l'indépendance politique est fondée sur une particularité culturelle. Le destin d'une nation n'est pas seulement l'indépendance, c'est aussi l'expression, le déploiement de cette originalité qui la fonde. 